06.1;06.3;12.2

\title{
Глубокая 3D-рентгенолитография на основе высококонтрастного рентгенорезиста
}

\author{
(C) В.П. Назьмов \\ Институт ядерной физики СО РАН, Новосибирск, Россия \\ E-mail: V.P.Nazmov@inp.nsk.su
}

Поступило в Редакцию 17 мая 2019 г.

В окончательной редакции 27 мая 2019 г.

Принято к публикации 3 июня 2019 г.

\begin{abstract}
В классической рентгеновской литографии рентгеновская маска и слой резиста располагаются перпендикулярно рентгеновскому лучу, который, поглощаясь в слое резиста, инициирует вдоль своего пути отклик в форме, соответствующей его поперечному сечению. Однако наклон и поворот маски/резиста, а также несколько экспозиций, проведенных последовательно, позволяют создать реальную трехмерную форму с точностью лучше микрометра. Описаны подходы к созданию реальных трехмерных микроструктур методом глубокой рентгеновской литографии, позволяющие формировать из них относительно большие массивы.
\end{abstract}

Ключевые слова: рентгеновская литография, двойное облучение, контрастность.

DOI: 10.21883/PJTF.2019.18.48227.17879

Различные трехмерные микроструктуры, например чешуйки самой быстрой известной в настоящее время акулы-мако [1], волокна на лапках геккона [2], микротекстура метаматериалов [3] и т.д., могут улучшить реальные свойства или придать новые существующим объектам в гидроаэродинамике, механике, электродинамике и других областях. Вследствие небольшого размера одной микроструктуры для получения определяющего физического эффекта требуется много подобных функциональных микроструктур. В отличие от стереолитографии [4], основанной на последовательном воздействии сфокусированного лазерного луча на каждый элементарный объем модифицируемого материала, метод трехмерной глубокой рентгеновской литографии, предлагаемый в настоящей работе, обеспечивает воспроизведение изображений, соответствующих размеру топологии на рентгеновской маске, которая может содержать много тысяч эталонных микроструктур. Ранее в работах по рентгенолитографии создание сложных форм базировалось либо на суперпозиции двумерных микроструктур [5], либо на вариации дозы, результатом чего явились примитивные трехмерные микроструктуры [6]. Предложенный вместо фотолитографии при изготовлении СБИС (сверхбольших интегральных схем) метод рентгеновской литографии [7] был позже развит до формирования микроструктур с высоким аспектным соотношением и широким спектром предназначений [8]. Сформированные с субмикронной точностью на большой площади массивы микроструктур могут быть предложены в качестве элементов устройств управления оптическим излучением и хранения информации [9], широкоапертурных рентгеновских линз [10] и т.д. Однако размер структур можно контролировать лишь в двух измерениях, что не позволяет изготавливать, например, микропризменные отражатели - катафоты или высокоэффективные антенные структуры фазированных антенных решеток [11]. Предложенный в настоящей работе подход позволяет формировать более сложные структуры на площади, обеспечиваемой шириной рентгеновского пучка синхротронного излучения (СИ) в несколько сантиметров.

Как и в классической рентгеновской литографии, перенос изображения осуществляется в режиме $1: 1$. Однако в отличие от воздействия одиночного рентгеновского луча в нашем исследовании сложное трехмерное распределение дозы формируется в слое резиста вследствие наложения дозовых распределений, создаваемых двумя лучами рентгеновского излучения, пересекающимися под углом $90^{\circ}$ друг к другу (рис. 1). Результирующее распределение дозы преобразуется в трехмерный профиль после жидкостного проявления высококонтрастного рентгеновского резиста mrx (производства компании MRT). Его высокая контрастность и высокое пространственное разрешение обусловлены эффективным сшиванием преполимерных молекул в пределах небольшого изменения дозы вблизи величины пороговой дозы [12].

Использование двухлучевого облучения позволило сформировать реальные трехмерные микроструктуры в слое резиста толщиной $200 \mu \mathrm{m}$ (рис. 2,a). Суммарная доза $D_{2}$ поглощенного в материале резиста излучения составила $36 \mathrm{~J} / \mathrm{cm}^{3}$, что соответствует длительности воздействия около 1 min для рентгеновских лучей, генерируемых источником СИ ВЭПП-3 для станции LIGA [13].

Чтобы свести к минимуму фоновую дозу $D_{1}$ в верхних слоях резиста, нами было испытано двустороннее облучение (рис. $1, b$ ): с тыльной стороны рентгеновский луч облучает слой резиста сквозь подложку, изготавливаемую из полиимидной фольги толщиной $25 \mu \mathrm{m}$. На рис. $2, b$ показан массив 3D-микроструктур пирамидальной формы (нижний уровень), сформированных двусторонним облучением. Поверх нижнего уровня сформиро- 

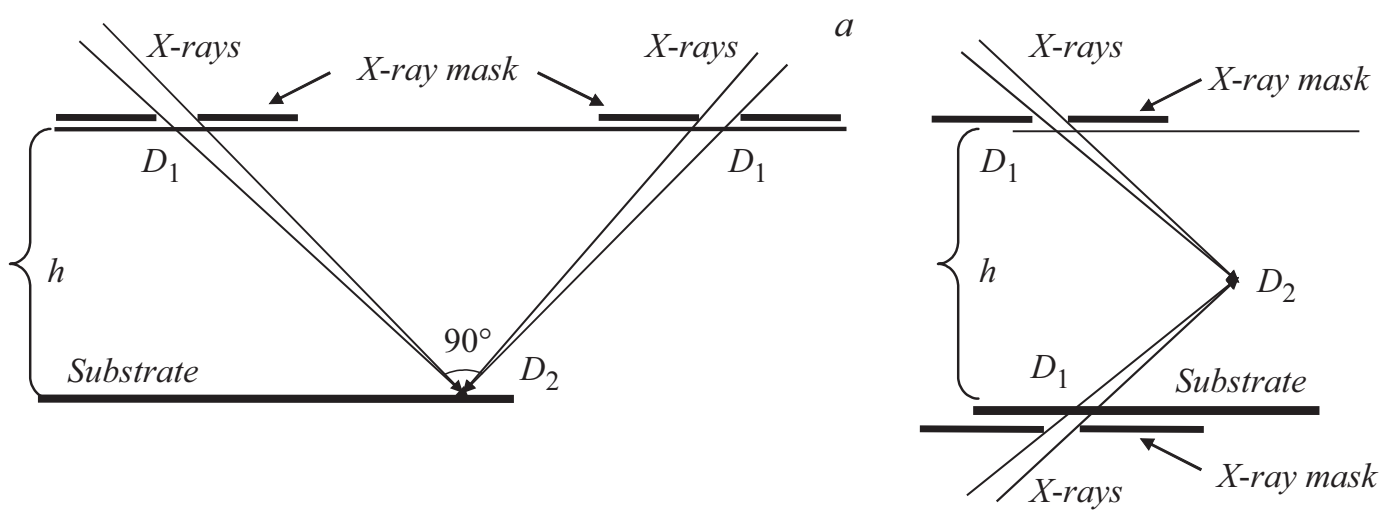

$b$

Рис. 1. Схема двухкратной экспозиции слоя резиста толщиной $h . a-$ с одной стороны, $b-\mathrm{c}$ двух сторон.

ван путем односторонних облучений верхний уровень, представляющий собой массив микротетраэдров.

В целях миниатюризации переносимое изображение рентгеновской маски может быть уменьшено с помощью
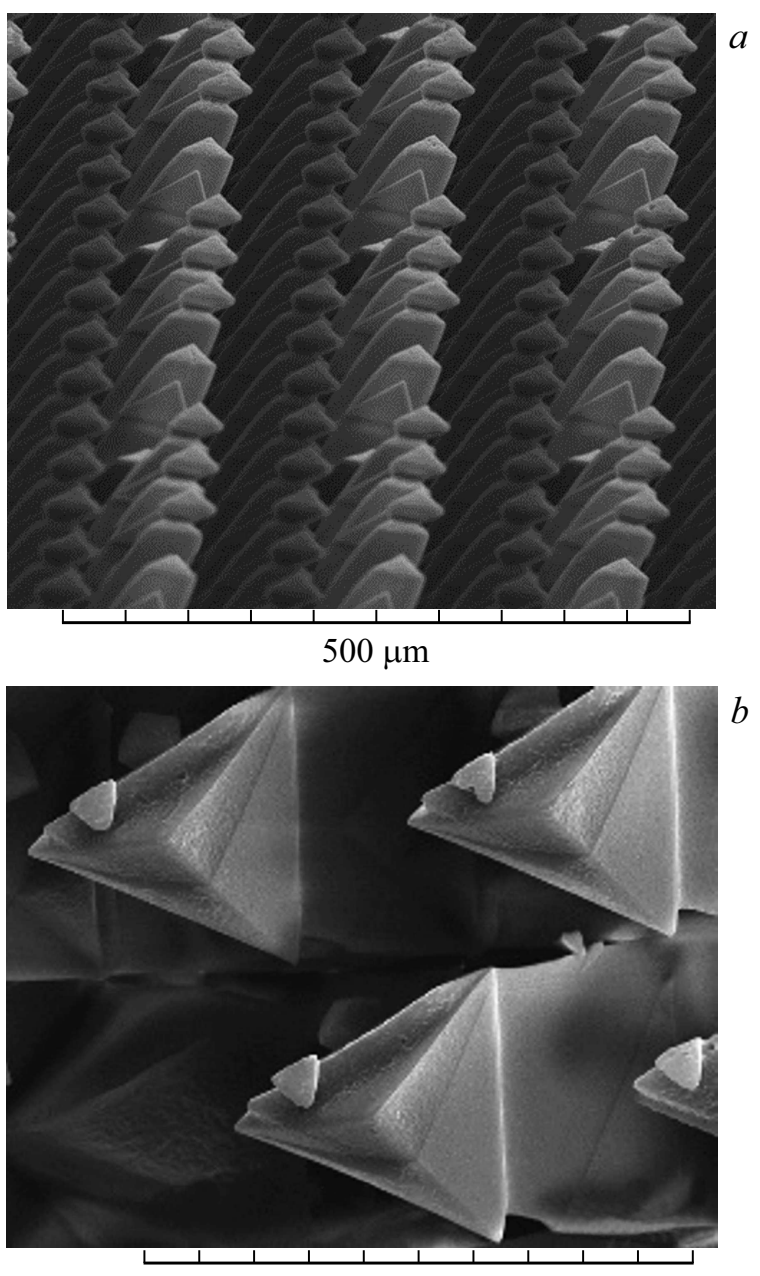

$100 \mu \mathrm{m}$

Рис. 2. 3D-микроструктуры, изготовленные в слое резиста $\operatorname{mrx}$ толщиной $200 \mu \mathrm{m} . a-$ зубчатые микроиглы, $b-$ двухуровневая микроструктура. преломляющей рентгеновской линзы [14], но поле зрения линзы составляет лишь десятки микрометров. Поэтому для переноса уменьшенного изображения элементов на площади, сравнимой с размером рентгеновской маски, маска помещалась на расстоянии $L \approx 0.33 A^{2} / \lambda$ от слоя резиста $(\lambda-$ длина волны, $A-$ размер топологического отверстия на рентгеновской маске), при котором наблюдается локальная концентрация освещенности вследствие дифракции рентгеновского излучения на краях топологических элементов маски, как предложено в [15]. Например, при монохроматическом когерентном освещении поперечный размер (FWHM) скрытого изображения за щелью шириной $A$ составляет $\sim 1.1 \lambda L / A$, что в 2.8 раза меньше размера на маске, а выигрыш в освещенности $G$ достигает величины 3.2 . Вдобавок поперечный размер элементов воспроизводимого изображения может быть уменьшен в $\gamma$ раз с учетом дозовой функции растворимости резиста, крутизна которой описывается параметром контрастности $\gamma$ [16].

Расчет минимального резистивного элемента был проведен для $A=900 \mathrm{~nm}, \lambda=2.5 \AA$ и $L=1070 \mu \mathrm{m}$. Несмотря на протяженную дозовую функцию (рис. $3, a$ ), боковые стенки моделируемого профиля близки к вертикальным. Чтобы минимизировать остатки резиста, генерируемые от сложения паразитных доз за пределами скрытого изображения актуального топологического элемента, величина контрастности резиста должна быть выше чем $\gamma=2.3 /[\ln (2 G)-1.41 \mu h]$, где $\mu-$ коэффициент линейного поглощения резиста на длине волны $\lambda$, а $h$ - толщина резистивного слоя (рис. $3, b)$. Минимальное значение контрастности уменьшается с увеличением длины волны вследствие более низкого коэффициента поглощения, а величина контрастности резиста mrx может достигать девяти [12].

Полученные массивы микроструктур могут быть использованы в качестве функциональных оптических, гидроаэродинамических и других элементов, а также матриц для последующего формирования функциональных слоев [17].

В дальнейшем использование рентгеновских масок с различной топологией позволит разнообразить результирующее скрытое изображение в слое резиста. 

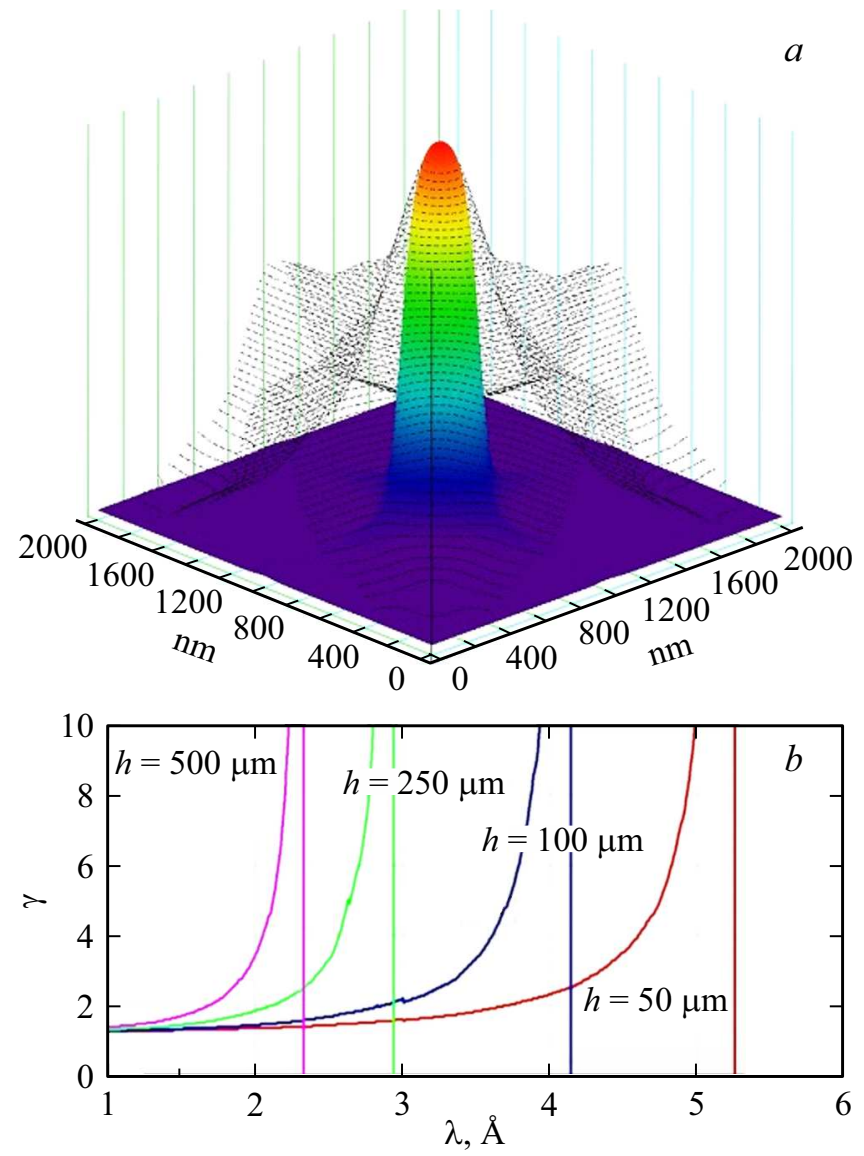

Рис. 3. $a-$ расчетное распределение дозы в материале резиста (с учетом диссипации энергии за счет фотоэлектронов) (пунктир) и профиль оставшегося резиста после удаления неполимеризованного материала (сплошная линия), $b$ - расчетный минимальный контраст в зависимости от длины волны рентгеновского излучения для различной толщины слоя резиста.

Следует отметить, что, поскольку радиационно-индуцированная температура влияет на чувствительность резиста [18], можно варьировать топологию формируемых трехмерных структур посредством задания мощности рентгеновских пучков.

Таким образом, в работе предложен метод изготовления с субмикронной точностью реальных трехмерных микроструктур высотой до нескольких сотен микрометров на площади до нескольких квадратных сантиметров с относительно высокой производительностью. Предлагаемый метод базируется на применении высококонтрастных рентгеночувствительных материалов.

\section{Благодарности}

Часть исследований, касающаяся экспонирования образцов, выполнена с использованием инфраструктуры Центра коллективного пользования „Сибирский синхротронный и терагерцевый радиационный центр“ на базе источника СИ ВЭПП-3 в Институте ядерной физики CO PAH.

\section{Финансирование работы}

Работа выполнена при финансовой поддержке Российского фонда фундаментальных исследований (грант 19-42-540014 p_a).

\section{Список литературы}

[1] Domel A., Saadat M., Weaver J., Haj-Hariri H., Bertoldi K., Lauder G. // J. R. Soc. Interface. 2018. V. 15. P. 20170828.

[2] Autumn K., Liang Y., Hsieh S., Zesch W., Chan W., Kenny T., Fearing R., Full R. // Nature 2000. V. 405. P. 681-685.

[3] Lu Y., Yang Y., Guest J., Srivastava A. // Sci. Rep. 2017. V. 7. P. 43407.

[4] Bertsch A., Renaud P. Microstereolithography // Threedimensional microfabrication using two-photon polymerization: fundamentals, technology, and applications / Ed. T. Baldacchini. Amsterdam: Elsevier, 2015. P. 47-71.

[5] Feiertag G., Ehrfeld W., Freimuth H., Kolle H., Lehr H., Schmidt M., Sigalas M.M., Soukoulis C.M., Kiriakidis G., Pedersen T., Kuhl J., Koenig W. // Appl. Phys. Lett. 1997. V. 71. P. 1441-1443.

[6] Horade M., Sugiyama S. // Microsyst. Technol. 2010. V. 16. P. 1331-1338.

[7] Spears D.L., Smith H.I. // Electron. Lett. 1972. V. 8. P. 102 104.

[8] LIGA and its applications, advanced micro \& nanosystems. V. 7 / Eds V. Saile, U. Wallrabe, O. Tabata, J. Korvink. Weinheim: WILEY-VCH GmbH, 2009. 480 p.

[9] Асадчиков В.Е., Бедин С.А., Васильев А.Б., Григорьев Ю.В., Назьмов В.П. // Письма в ЖТФ. 2019. Т. 45. B. 5. C. $49-51$.

[10] Simon M., Reznikova E., Nazmov V., Last A., Jark W. // Proc. SPIE. 2008. V. 7077. P. 70771Q. DOI: $10.1117 / 12.795423$

[11] Фёдоров С.М. Сверхширокополосные линзовые антенны с коммутационным сканированием в азимутальной плоскости. Канд. дис. Воронеж: Воронеж. гос. техн. ун-т, 2013. $146 \mathrm{c}$.

[12] Kunka D., Mohr J., Nazmov V., Meiser J., Meyer P., Amberger M., Koch F., Schulz J., Walter M., Duttenhofer T., Voigt A., Ahrens G., Grützner G. // Microsyst. Technol. 2014. V. 20. P. 2023-2029.

[13] Гольденберг Б.Г., Лемзяков А.Г., Зелинский А.Г., Назьмов В.П., Пиндюрин В.Ф. // Поверхность. Рентгеновские, синхротронные и нейтронные исследования. 2016. № 1. C. 64-67.

[14] Schroer C., Benner B., Güzler T., Kuhlmann M., Zimprich C., Lengeler B., Rau C., Weitkamp T., Snigirev A., Snigireva I., Appenzeller J. // Rev. Sci. Instrum. 2002. V. 73. P. 1640-1642.

[15] Bourdillon A., Boothroyd C., Williams G., Vladimirsky Y. // J. Phys. D: Appl. Phys. 2003. V. 36. P. 2471-2482.

[16] Валиев К.A. // Физика субмикронной литографии. М.: Наука, 1990. 528 c.

[17] Schaedler T.A., Jacobsen A.J., Torrents A., Sorensen A.E., Lian J., Greer J.R., Valdevit L., Carter W.B. // Science. 2011. V. 334. P. 962-965. DOI: 10.1126/science.1211649

[18] Назьмов В., Бергер М., Хайслер С. // Поверхность. Рентгеновские, синхротронные и нейтронные исследования. 2011. № 11. C. $3-7$. 